\title{
物种濒危状态等级评价概述
}

\author{
成克武* 蔵润国 \\ (中国林业科学研究院森林生态环境与保护研究所, 北京 100091)
}

\begin{abstract}
摘要: 濒危等级是确定物种优先保护顺序和制订濒危物种保育策略的重要依据，《生物多样性公约》、相关国际组 织和一些国家都把物种濒危状态的评价作为生物多样性保护工作中的一个重要步骤。本文对 IUCN 和美国、加拿 大等国在物种濒危状态评价中有关等级标准、评价程序、数据信息采集管理及网络应用方面的问题进行了分析。 国际上物种濒危状态评价工作呈现出以下几个方面的发展趋势: (1) 物种濒危等级评价标准由定性指标向定量指 标发展; (2)物种濒危状况评价程序逐步规范严格，评价过程透明，公众积极参与;(3) 数据信息的采集逐步标准化， 储存与管理更新采用计算机及网络技术; (4)物种濒危等级的公布与更新逐步以网络形式为主; (5) 全球对物种濒 危状态评价工作日益重视，物种濒危等级信息在物种资源利用、保护及其他方面的应用愈加广泛。在此基础上，作 者提出了我国在物种濒危状况评价中急需加强的 3 个方面的工作: (1) 建立我国的物种濒危等级评价标准; (2) 建 立完善的物种濒危等级评价程序并制定相关的指导性文件; ; (3) 加强濒危物种评价中数据信息的规范采集、网络管 理和数据共享。
\end{abstract}

关键词: 物种, 濒危等级, 评价,世界自然保护联盟

中图分类号 : Q16 文献标识码 : A 文章编号 : 1005-0094(2004)05-0534-07

\section{Advances in species endangerment assessment}

CHENG Ke-Wu* , ZANG Run-Guo

The Research Institute of Forest Ecology , Environment and Protection, Chinese Academy of Forestry , Beijing 100091

\begin{abstract}
The category of endangerment is an important reference for determining conservation priority of rare and endangered species. The assessment of endangerment status is considered to be one of the most important steps in biodiversity conservation by the Convention on Biological Diversity , related international organizations and many countries. This paper introduces the selection criteria for species endangerment assessment , assessment procedure , information collection, and the application of Internet technology in species endangerment assessment. Based on the above, the development trends in the endangerment assessment include : (1) the assessment criteria are developing from qualitative to quantitative ones ; $(2)$ the assesement procedures are becoming more and more formal and open ; 3 ) data collection is becoming more and more standardized, and the information storage and processing further computerized ; (4) species endangerment criteria are published and updated mainly through Internet, and (5) species endangerment assessment is attracting international attention, and it is widely used in the conservation and management of wildlife resources. At last, we put forward some suggestions on the species endangerment assessment in China : (1) establishing species endangerment criteria ; (2) setting up assessment procedure and related regulations ; (3) standardizing data collection, improving data management on the Internet and data sharing.
\end{abstract}

Key words : species , endangerment category , assessment , IUCN

濒危物种是生物多样性的重要组成部分,加强 濒危物种的保护对于促进生物多样性的保护具有重
要意义。2002 年 4 月在海牙举行的《生物多样性公 约》缔约方大会第六次会议上提出了全球生物多样 
性保护策略的 16 个目标, 其中的第二项内容就是开 展在国家、地区和全球范围内的物种濒危等级评价 工作 (CBD 2004)。目前, 许多国家和一些相关的国 际组织都在积极开展物种评价工作。但是，各国对 濒危物种的定义不同，所应用的评价标准、方法和程 序也不同，这直接影响到物种濒危等级的确定和濒 危物种的保护。分析和总结国际上的先进经验，对 于促进我国物种的濒危等级评价和保护工作具有很 好的借鉴作用。本文在分析世界自然保护联盟 (The World Conservation Union，IUCN) 和美国、加拿 大等国物种濒危等级评价标准、方法及评价程序的 基础上, 提出我国需要加强和改进的方面。

\section{1 濒危物种、濒危等级的概念及其与物种保 护之间的关系}

濒危物种 (endangered species) 通常是指由于物 种自身原因或受到人类活动或自然灾害影响而有灭 绝危险的所有生物种类 (李鹏翔, 2002)。如《濒危 野生动植物物种国际贸易公约》 ( the Convention on

International Trade in Endangered Species of Wild Fauna and Flora，CIETS) 附录中所列的濒危物种是 指由于国际贸易而可能灭绝的物种; 而在物种濒危 等级评价标准中的濒危物种, 则是依据物种灭绝危 险程度而划分的。由于 IUCN 及许多国家、地区各 自制订的濒危等级体系不同，体系中各个等级类型 的评价指标不同，造成了在不同体系中濒危物种具 有不同的概念和评价指标: (1) 在 IUCN 颁布的最 新的《IUCN 红色名录等级标准》3.1 版 ( 以下简称 IUCN 标准) 中 ,对 濒危物种”的定义为 :还没有达 到极危等级，但其野生种群在不久的将来面临很高 的灭绝风险，符合 濒危” 等级 5 项量化指标之一的 物种 (IUCN，2001)；(2) 美国的物种濒危等级体系 中 将濒危物种定义为 在目前或不远的将来, 在其 分布区范围的全部或重要组成部分内面临灭绝危险 的动植物物种”，采用了定性的评价指标( FWS , 2002 ); (3) 加拿大将濒危物种定义为 在 (加拿大) 全国或全球范围即将面临灭绝的物种”，其量化指 标范围包括了 IUCN 标准中 濒危” 和 极危” 两个 等级的评价指标范围 (COSEWIC , 2003);(4) 澳大利 亚关于濒危物种的定义与 IUCN 早期定性的濒危等 级体系中 濒危” 等级的含义相近, 但物种范围仅限 于澳大利亚土著种 ( SCALEplus ,2000;SCALEplus ，
$1999) ;(5)$ 《中国植物红皮书》对濒危物种的定义 为 物种在其分布的全部或显著范围内随时有灭绝 的危险, 通常生长稀疏, 个体数和种群数低, 且分布 高度狭域;由于栖息地丧失、破坏或过度开采等原 因，其生存濒危（傅立国，1992 ; 蒋志刚和樊恩源， 2003 )。

应用不同的评价指标对物种的濒危程度 (即灭 绝概率大小) 进行划分, 就构成了物种的濒危等级。 各个国家 (或组织、地区) 对物种濒危等级的划分方 法和指标不同，从而形成了各自的划分体系和等级 名称。如 IUCN 标准中, 将全球物种的濒危等级划 分为灭绝、野外灭绝、极危、濒危、易危、近危、无危、 数据缺乏、未评估 9 个级别, 而在《IUCN 红色名录 标准在地区水平的应用指南》3.0 版 (Guidelines for Application of IUCN Red List Criteria at Regional Levels：Version 3.0) 中，对一个国家或地区内物种的濒 危等级共划分为 11 个:灭绝、野外灭绝、地区灭绝、 极危、濒危、易危、近危、无危、数据缺乏、不适用、未 评估( IUCN ,2003a)。美国鱼类与野生生物署( U. S. Fish and Wildlife Service, FWS) 将美国的物种划 分为濒危、受威胁两个等级类型 (FWS ,1988) ; 加拿 大野生物种濒危状态委员会 (Committee on the Status of Endangered Wildlife in Canada ,COSEWIC) 参 照 IUCN 标准制订的物种濒危等级包括灭绝、国内 灭绝、濒危、受威胁、特别关注、数据缺乏、无危共 7 个级别( COSEWIC ,2003) ;澳大利亚受威胁物种等 级类型包括: 灭绝、野外灭绝、极危、濒危、易危、依赖 保护 6 个类型 (SCALEplus ,2000)。我国在 1992 年 《中国植物红皮书》中参考 IUCN 标准, 采用了濒危、 稀有、渐危三个等级 (傅立国 ,1992)。由此可以看 出，在物种濒危等级划分方面全球并没有一个统一 的等级体系。相比之下, IUCN 标准的等级体系中各 濒危等级类型最全面，其他国家则参差不齐。随着 计算机技术、网络技术和数据信息存储管理技术的 发展 建立完善的等级体系将是各国制订物种濒危 等级标准的大趋势。

物种濒危状态的评定对于物种的保护具有重要 意义。由于自然界的生物种类极为丰富，对每一个 物种都采取同样的保护措施显然是不可能的，如何 针对处于不同濒危状态的物种采取不同的保护策 略, 使濒危物种得到有效保护, 最大程度地延缓物种 的灭绝速度, 是生物多样性保护工作中很关键的问 
题。通过对物种濒危状况的评价，确定物种的优先 保护顺序，有针对性地采取合理有效的保护措施，才 能使有限的人力、物力资源得到最佳配置和发挥, 使 真正濒危的物种得到及时的保护（崔国发等， 2000 )。

但是，物种的濒危等级只是确定优先保护顺序 和制定保护策略的影响因素之一, 其他因素如经费 开支、现有的技术水平、物种保护的成功几率、该物 种的分类学地位等也是需要考虑的方面 ( IUCN, 2001 ;Gärdenfors et al. ,2001)。

\section{2 物种濒危状态评价}

\section{1 评价的历史与现状}

对物种濒危等级的划分最早开始于 20 世纪 60 年代的 IUCN 濒危物种红皮书, 该书中关于物种濒 危等级的划分得到了一些国家的采用 (Nilsson， 1990 ; 解炎和汪松，1995; 蒋志刚和樊恩源，2003）。 此后, IUCN 通过不断修改和更新, 形成了目前相对 稳定的标准。目前, 许多国家还没有制订相应的物 种濒危等级评价标准, 更不用说规范的评价过程; 有 些国家即使制订了评价标准，但标准中各等级的划 分仍依据定性指标，给评价工作带来不便，使得评价 结果受人为因素影响较大; 有些国家虽然制订了相 应的评价标准和指标，但评价程序不规范，评价的结 果也受到影响; 另有些国家如美国已经制定了严格 的评价标准和评价程序, 评价过程规范严谨, 但评价 过程相对较长、费用较高。

我国近年来对濒危物种的保护力度不断加强， 对濒危物种的评价方法、程序都给予了高度的重视， 但是由于我国在这方面的工作起步较晚，与发达国 家仍存在一定的差距。目前, 针对林业行业的森林 植物物种濒危等级评价标准正在制订之中; 在动物 方面，汪松、郑光美等人参照 IUCN 标准，出版了我 国濒危动物红皮书兽类、鸟类、鱼类和两栖爬行类卷 (郑光美和王岐山,1998; 汪松, 1998; 乐佩琦和陈 宜瑜，1998；赵尔宓，1998），从长远来看，制订并明 确中国的动物濒危等级标准，有利于中国动物的濒 危状况评价及保护工作。

2.2 濒危等级评价方法与评价指标的选择

由于受地理条件、研究水平、经济力量等方面的 影响，许多国家、地区以及 IUCN 等对物种的濒危等 级的评价方法和指标的选择各不相同。IUCN 的濒
危等级从 1991 年开始采用量化评价指标后 到目前 发布的 3.1 版 经历了多次修改。IUCN 3.1 版的几 个主要指标包括 种群减少情况、分布区与占有面积 变化情况、种群内成熟个体数量及其变化情况、估计 的种群成熟个体数以及定量分析预测今后一个时期 内物种的灭绝速率共 5 个方面 ( IUCN 2001)。美国 的濒危物种等级标准根据 (1) 物种生境/范围的破 坏、调整、缩减; (2) 以商业、娱乐、科研、教育为目的 的过度利用; ; 3 ) 疾病或掠夺; (4) 现有法规及管理 机制不完备; (5) 其他自然或人为影响因素等 5 个 方面对物种濒危等级进行确定 (FWS ,2001)。虽然 其标准没有确定各个级别评价的具体数量指标, 但 其评价过程采用了“候选物种”、优先评估”和 公 众评议”制度, 并且有规范的物种信息数据和严格 的评价程序作保障, 确保了评价结果的透明度和公 众可接受度。加拿大不列颠哥伦比亚省可持续资源 管理部 (Ministry of Sustainable Resource Management)下属的保育数据中心 ( Conservation Data Center , CDC) 采用 NatureServe 建立的全球物种状态等 级体系中，对各个等级的划分主要以在全球出现的 地点数、个体数或面积作为划分依据（WLAP， 2002 ) 英国联合自然保育委员会 (Joint Nature Conservation Committee JNCC) 1995 年对英国稀有植物 划分的两个类型 红皮书物种" (Red Data Book Species) 和 国家稀有物种” ( Nationally Scarce Species) 中 采用了物种出现在 10 公里方格网中的数量作为 评价指标 (Palmer ,1995)。在 JNCC 目前正在推广 应用的物种濒危等级系统中，两个等级 国家稀有” ( Nationally Rare ,NR) 和“国家偶见”（ Nationally Scarce ,NS) 也采用了在全国的分布面积作为评价 指标 ( JNCC 2003)。

尽管目前国际上对 IUCN 标准在一些分类单元 上的适用性以及某些指标的阈值设置方面仍然存在 争议,但是, 该标准是在广泛征求意见, 并经过多次 修改和实地测试，得到最广泛专家认可基础上形成 的评价体系，已经被英国、加拿大、新加坡等许多国 家部分采用，其他一些国家的标准制订也在向 IUCN 标准靠近。这一趋势将有助于不同国家间的交流和 不同分类单元物种的濒危状况之间的比较，也有助 于对全球生物多样性濒危状况的了解。

\section{3 物种濒危等级的评估申请}

IUCN 下属的物种生存委员会 ( Species Survival 
Council, SSC) 通过在网上提供红色名录调查表 (Red List Questionnaire) 的方式, 寻求公众对红色名 录专家组成员的帮助 (主要是征求意见与信息)，并 且为公众提出的红色名录评估的申请提供服务。该 调查表包含了濒危等级评估所需的相关信息和权威 性文件，尤其是关于物种的生境、所受威胁、保护行 动和利用状况方面的内容, 通过附带的权威性文件 进行了详细的规范和标准化( IUCN ,2003b)。申请 者可通过网络将调查表递交给红色名录项目组。

在美国，根据《濒危物种法》( Endangered Species Act，ESA) 规定，一个物种可以通过两种不同的 提交途径进行评估，即通过申请程序和候选物种评 价过程，以决定是否可以被列入联邦濒危与受威胁 物种名录中 (Nicholopouios，2003)。任何感兴趣的 人都可以向美国内务部申请从濒危与受威胁物种名 录中增加或去除一个物种; 候选物种评价过程是指 美国鱼类与野生生物署的生物学家按照优先顺序对 候选物种进行濒危等级评价的过程。而候选物种 (Candidate Species) 是指美国鱼类与野生生物署拥 有足够的有关该物种的生物学特性及所受威胁的信 息，并依据《濒危物种法》提议对它们进行濒危与受 威胁等级评价, 但是根据提议规则, 由于其他更重要 物种的优先评价行动而被排除的动植物物种。除上 述两种评价申请方式, 美国还有一项紧急预案 :即有 证据表明某个物种存在重大灭绝危险时, 则需要根 据濒危等级紧急列入程序进行评估。

与美国相似，加拿大也拟定了相应的物种濒危 等级评价申请表及相关的申请程序, 通过招标方式 从签约人手里获取要评价物种的濒危状态报告 (FWS ,1988)。

\section{4 物种濒危等级的评价过程}

物种濒危等级的评价过程一般是指由主管部门 (或组织) 成立专门的评价委员会, 负责对各物种濒 危等级的申请报告进行科学评议，由委员会提出决 策性建议与意见。评价过程的主要环节包括评价过 程中相关数据信息的获取、补充与验证，公众的评议 与意见反馈，以及最终由专家做出的评价结果。

IUCN 标准及其应用指南对物种评价中所需数 据资料的类型与范围、获取方法及评价过程等都有 明确的规定。专家需要对提交物种的分类学地位、 分布信息、种群动态、濒危状态、致危因素、保护措 施、数据来源、分布图、列入濒危等级的理由等方面
的信息及各数据的处理过程、推导公式和相关环节 的处理进行分析研究( IUCN 2001)。新物种列入前 必须经过 IUCN 红色名录权威组两名专家的评价, 评价过程必须遵守 SSC 的规定 (IUCN，2001， 2003 c)。

美国《濒危物种法》对本国物种濒危等级的评 价过程的规定也极为严格，从申请的提出到最后通 过, 都有一系列的法定程序, 在评价过程中接受社会 各方面的质询。美国物种濒危状态评价的流程如图 1 所示 (Nicholopouios, 2003)。

加拿大濒危物种的评价, 是由加拿大濒危物种 保护委员会 (the Canadian Endangered Species Conservation Council ,CESCC) 提供评委人员组成、评议 程序及评价标准方面的指导, 通过加拿大野生物种 濒危状态委员会 (COSEWIC) 来完成。COSEWIC 评 委会人员的组成和任命都按照相关的法律程序确 定,包括保护生物学、生态学、分类学、野生物种管 理、价值评估、种群生物学、群落学及相关领域的专 家, 评价范围包括任何本土的种、亚种、变种,或地 理、遗传基因独特的野生动植物种群。COSEWIC 的 评价过程分为 3 个连续的步骤, 通过每一个步骤都 要达到一个具体的目标。这三个步骤分别为:(1) 物种的选择及其优先顺序一确定优先的候选物种 名录; (2) 有效数据、知识及信息的收集汇编一完 成 COSEWIC 状态报告。状态报告包含有关评议物 种的生物学特性、在加拿大的种群大小和分布状况、 种群变化趋势、生境可利用性。在 COSEWIC 评估 会议上,评估报告将作为讨论与初步确定物种濒危 状态的基础; (3) 物种灭绝风险评价及其濒危等级 的初步确定一COSEWIC 关于物种濒危状态等级 的初步确认 (COSEWIC, 2003)。可以看出, 规范的 评价程序是科学开展物种濒危状况评价工作的重要 保障。

\section{5 物种濒危等级的确定与等级变化}

专家委员会对申请报告作出的评价结果，只有 通过主管者(部门)的最终通过并予以公布,才能最 终确定物种的濒危等级。由于濒危等级的评价过程 一般是由评价委员会负责，而濒危等级的最终确定 由主管者决定，因此二者是相对独立的。

为了使更多的使用者更方便地获取有关信息， 从 2000 年开始, IUCN 红色名录除了书籍形式外, 开 始以电子媒体的形式在网上公布。该名录每年更新 


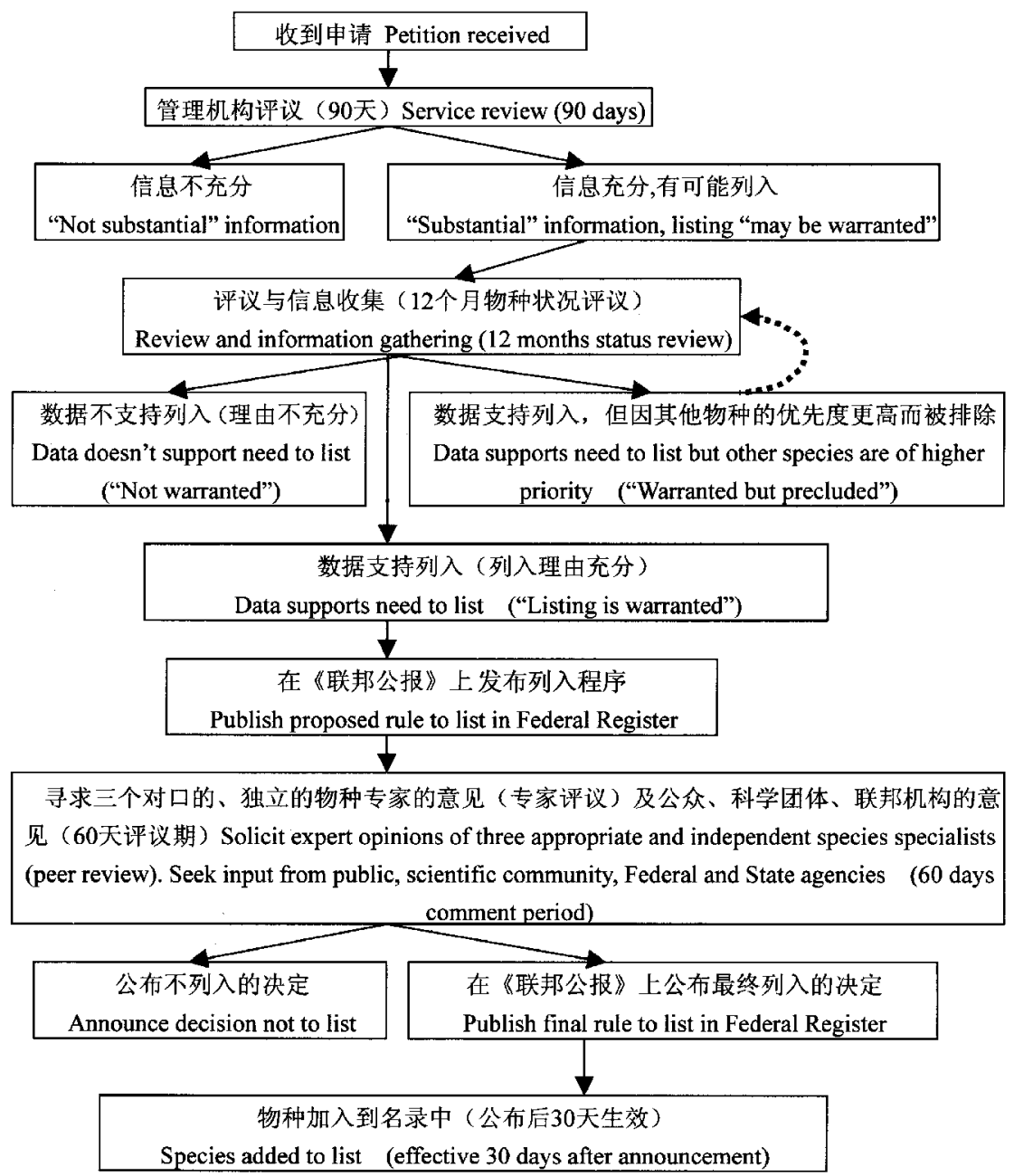

图 1 美国物种濒危状态评价流程图 ( 引自 Nicholopouios , 2003)

Fig. 1 Flow chart of species endangerment assessment in the U. S. A. (Cited from Nicholopouios ,2003)

一次, IUCN 下属的物种生存委员会红色名录项目 ( SSC Red List Program)办公室负责维护并提供各物 种的相关数据信息( IUCN 2003d)。IUCN 标准对列 入红色名录中的物种的濒危等级随数量、生境等的 变化作了明确规定，一般要求 5 到 10 年重新评估一 次。引用该标准中物种的濒危等级时 须注明评价 所用标准的版本号以及该物种所符合的濒危标准条 款。

美国物种濒危等级的最终确定要通过《联邦公 报》(Federal Register) 进行公布。对于列入濒危与 受威胁物种名录的物种，必须包含以下信息 濒危等 级、被确定为该等级的具体日期、分布区内的濒危范 围、已知及主要的分布地区、提议列入和最终列入濒 危等级名录的相关文件等 (FWS , 2004)。加拿大和
其他一些国家也对濒危等级的变化作出了明确规 定, 并在网上发布相关信息。

\section{6 物种濒危状态评价的发展趋势}

从国际上对物种濒危等级评价的发展趋势来 看, 主要表现出以下特点: (1) 物种濒危等级评价标 准由定性指标向定量指标发展（崔国发等，2000）； (2)物种濒危状况的评价程序逐步规范严格，评价 过程透明，公众积极参与;(3) 数据信息的采集逐步 标准化，其存储及管理更新采用计算机技术; (4) 物 种濒危等级的公布与更新管理逐步以网络形式为 主; (5) 全球对物种濒危状态评价工作日益重视, 物 种濒危等级信息在物种资源利用、保护及其他方面 应用更加广泛。 


\section{3 我国的濒危物种评价工作}

我国从 20 世纪 80 年代才开始物种濒危等级评 价方面的研究工作。由于起步晚, 有关动植物濒危 等级评定标准体系还没有正式出台，相关的政策法 规、管理体制和专家库建设还不健全，物种濒危等级 的评价程序和数据共享机制还不完备，这些因素都 制约了我国的物种濒危状态评价，从而影响到我国 濒危物种的有效保护。参照 IUCN 和美国、加拿大 等国的经验与方法, 我国的濒危物种评价工作应该 在以下领域予以加强 :

\section{1 建立我国的物种濒危等级评价标准}

IUCN 标准主要应用于全球范围内的物种濒危 状况评定，在国家、地区范围的应用还存在一些问 题; 同时，由于各个国家的科技水平和管理体制不 同 完全采用 IUCN 标准是不可能的。但是该标准 中关于“极危、濒危、易危” 等级的量化指标已经被 广泛接受, 这一标准也正在被英国、加拿大、澳大利 亚、新加坡等国所部分采用。我国在制订本国的濒 危物种评价标准时, 应充分吸收相关的成果和经验, 使我国濒危物种的等级评价建立在量化的基础上。

3.2 建立完善的物种濒危等级评价程序和制定相 关的指导文件

从 IUCN 和美国、加拿大等国的濒危物种评价 过程来看, 制订规范的物种评价程序及配套的规章 制度，建立包括分类学、生态学、保护生物学等相关 领域的专家库 (尤其是包括专属、专科领域的研究 专家和地方一级的专家、科研及保护人员信息库)， 增加评价过程的透明度并广泛吸收各方面的意见和 信息反馈，制订有关数据采集、关键指标阈值确定、 评估报告内容等方面的规范性文件，以招标、签约或 其他方式鼓励专家、地方保护机构或感兴趣者提出 有关物种的濒危状态评价申请, 能够有力促进物种 濒危状态评价工作的开展和相关数据信息的采集。 我国在开展濒危物种评价工作中, 应该充分借鉴吸 收国外的先进经验，并根据我国的实际情况和未来 发展需要, 建立健全各项评价程序与制度。

3.3 加强濒危物种数据信息的采集、网络管理和数 据共享

IUCN 和美国、加拿大等国对列入红色名录的物 种一般每隔 5 到 10 年重新评估一次，以适应不断变 化的物种濒危状态。对于物种信息的收集与存储管
理, 美国、加拿大等国通过构建全国网络数据库, 依 靠地方管理部门对当地濒危物种信息的变动进行维 护，可以使全国的物种信息每 3 个月更新一次。对 于列入国家红色名录的物种，通过网上公布，可以使 更多的使用者获取。同时，根据物种濒危状态的评 价结果或增减情况，对网上公布的红色名录每年更 新一次。网络化的数据信息为上述工作提供了可 能。我国的物种濒危状况评价在数据收集和更新、 定期评估以及网络管理方面, 还有待进一步加强。

总之，科学的物种濒危等级评价是确定物种优 先保育顺序以及制定各物种保护策略的重要依据， 加强物种的濒危评价工作将有力地促进我国濒危物 种的保护。

\section{参考文献}

CBD. 2004. Global strategy for plant conservation. http:// www. biodiv. org $/$ decisions $/$ default. aspx? $\lg =0 \& p r i n t=$ $1 \& \mathrm{dec}=\mathrm{VI} / 9$.

COSEWIC. 2003. COSEWIC's assessment process and criteria. http: //www. cosewic. gc. ca/eng/sct0/Assessment_process tbl6_e. cfm.

Cui, G. F. (崔国发), Cheng, K. W. (成克武)，Lu，D. Z. (路 端正), Wang, J. Z. (王建中) and Li, J. Q. (李俊清).

2000. Evaluation on threatened situation and protection classes of vegetation in Beijing Labagoumen Reserve. Journal of Beijing Forestry University (北京林业大学学报), 22(4) : 8 -13. (in Chinese with English abstract)

Fu，L. G. (傅立国). 1992. China Plant Red Data Book: Rare and Endangered Plants. Science Press, Beijing \& New York, 741.

FWS. 1988. Endangered species act of 1973. http://endangered. fws. gov/esaall. pdf.

FWS. 2001. The endangered species act of 1973. http://endangered. fws. gov/esa. html\#Lnk04.

FWS. 2002. Endangered species glossary. http://midwest. fws. gov/endangered/glossary/index. html.

FWS. 2004. Species information: threatened and endangered animals and plants. http: //endangered. fws. gov/wildlife. html \#Species.

Gärdenfors, U., Hilton-Taylor, C. , Mace, G. and Rodríguez, J. P. 2001. The application of IUCN Red List Criteria at regional levels. Conservation Biology, 15: 1206 - 1212.

IUCN. 2001. IUCN Red List Categories and Criteria (Version 3. 1). IUCN Species Survival Commission. IUCN, Gland, Switzerland and Cambridge, UK.

IUCN. 2003a. Guidelines for Application of IUCN Red List Crite- 
ria at Regional Levels (Version 3.0). IUCN Species Survival Commission. IUCN, Gland, Switzerland and Cambridge, UK.

IUCN. 2003b. Red list assessment questionnaire. http: //iucn. org/webfiles/doc/SSC/RedList/AuthorityF/questionnaire. rtf.

IUCN. 2003c. The SSC Red List Program. http: //www. redlist. org/info/programme. html.

IUCN. 2003d. The IUCN Red List of threatened species. Organization of Information. http: //www. redlist. org/info/organization. html.

Jiang, Z. G. (蒋志刚) and Fan, E. Y. (㚞恩源). 2003. Exploring the endangered species criteria: rethinking the IUCN Red List Criteria. Biodiversity Science (生物多样性), 11: 383 - 392. (in Chinese with English abstract)

JNCC. 2003. Threatened, Rare and Scarce Plant Species. http: //www. jncc. gov. uk/species/Plants/threatened/default. htm.

Le, P. Q. (乐佩琦) and Chen, Y. Y. (陈宜瑜). 1998. China Red Data Book of Endangered Animals: Fish(中国濒危动 物红皮书口鱼类). Science Press, Beijing. (in Chinese with English translation)

Li，P.X. (李鹏翔). 2002. Do you know what is endangered species? Gansu Forestry (甘肃林业), (5)：37. (in Chinese)

Nicholopouios, J. 2003. The Endangered Species Listing Program. http://endangered. fws. gov/ESB/99/11-12/6-9. pdf.
Nilsson, G. 1990. The Endangered Species Handbook. The Animal Welfare Institute, Washington, D. C.

Palmer, M. 1995. A UK Plant Conservation Strategy. Joint Nature Conservation Committee, Peterborough.

SCALEplus. 1999. Environment protection and biodiversity conservation act 1999-Sect 179 Categories of threatened species. http://scaleplus. law. gov. au/html/pasteact/3/ 3295/0/PA003120. htm

SCALEplus. 2000. Environment protection and biodiversity conservation regulations 2000 - REG 7.01 Criteria for listing threatened species. http://scaleplus. law. gov. au/html/ pastereg/3/1619/0/PR000390. htm.

Wang, S. (汪松). 1998. China Red Data Book of Endangered Animals: Mammalia (中国濒危动物红皮书口兽类). Science Press, Beijing. (in Chinese)

WLAP. 2002. Species ranking in British Columbia. http:// wlapwww. gov. bc. ca/wld/documents/ranking. pdf.

Xie, Y. (解炎) and Wang, S. (汪松). 1995. IUCN Red List Categories. Chinese Biodiversity (生物多样性), 3: 234 239. (in Chinese)

Zhao, E. M. (赵尔宓). 1998. China Red Data Book of Endangered Animals: Amphibian \& Reptile (中国濒危动物红皮 书口两栖爬行类). Science Press, Beijing. (in Chinese)

Zheng, G. M. (郑光美) and Wang, Q. S. (王岐山). 1998. China Red Data Book of Endangered Animals: Aves(中国 濒危动物红皮书口鸟类). Science Press, Beijing. (in Chinese) 